\title{
Light ion beams for ADS energy production
}

\author{
A. A. Baldin ${ }^{1,2, *}$, A. I. Berlev ${ }^{1,2}, M$. Paraipan ${ }^{1,3}$, and $S . I$. Tyutyunnikov ${ }^{1}$ \\ ${ }^{1}$ Joint Institute for Nuclear Research, Dubna, 141980 Russia \\ ${ }^{2}$ Institute for Advanced Studies "OMEGA", Dubna, 141986 Russia \\ ${ }^{3}$ Institute of Space Science, Bucharest-Magurele, 077125 Romania
}

\begin{abstract}
The comparative analysis and optimization of the energy efficiency for proton and ion beams in ADS systems is performed via GEANT4 simulation with account of energy consumption for different accelerator types. It is demonstrated that for light nuclei, beginning from ${ }^{7} \mathrm{Li}$, with energies above $1 \mathrm{GeV} /$ nucleon ion beams are substantially more efficient than $1-3 \mathrm{GeV}$ proton beams.
\end{abstract}

\section{Introduction}

The application of accelerated beams for power production has been discussed since $1950^{\text {se }}[1]$. The concept of an energy amplifier implies neutron flux generation at interaction of an extracted beam with a target and energy production based on ${ }^{238} \mathrm{U}$, thorium ${ }^{232} \mathrm{Th}$, and other heavy elements fission.

ADS systems possess a number of advantages, such as the possibility of afterburning (transmutation) of radioactive waste (minor actinides), the shorter, as compared to fast reactors, fissile materials production cycle, and so on [2-5]. With increasing beam energy, and correspondingly, hardening the secondary neutron spectrum, it may become possible to use spent nuclear fuel as the fission source [6, 7].

Long-term theoretical and experimental studies formed a widely spread opinion that ADS-optimal beams are protons with an energy from $500 \mathrm{MeV}$ to $1.5 \mathrm{GeV}[4,5,9]$. The commonly considered concept of ADS energy production [10] assumes that a high current proton beam with an energy of $\sim 1 \mathrm{GeV}$ and a target station of heavy elements (natural uranium, thorium) provides an energy amplification factor of 30-40, which makes the development of an energy producing installation realistic. Recent experimental studies [7, 14] with deuteron beams, however, as well as the results of simulation demonstrate that the proton beam energy for $\mathrm{ADS}$ is higher, in a range of 2-4 GeV [12]. Since the energy required for accelerating a beam in an accelerator grows quadratically with the beam energy, it is necessary to optimize the beam taking into account the energy spent for the accelerator maintenance.

The possibility of replacing proton beams by light ion beams has been studied insufficiently. Thus, it was stated in $[10,11]$ that ions with a mass higher than that of tritium are less efficient for ADS. In [8], on the contrary, it was demonstrated that the most

${ }^{*}$ Corresponding author: an.baldin@mail.ru 
efficient is the relativistic ${ }^{20} \mathrm{Ne}$ nuclear beam with an energy of $\sim 3 \mathrm{GeV} /$ nucleon, which agrees with our preliminary results [16].

\section{Simulation}

The interaction of protons and ions (deuterons, tritium nuclei, ${ }^{7} \mathrm{Li},{ }^{9} \mathrm{Be},{ }^{11} \mathrm{~B},{ }^{12} \mathrm{C},{ }^{14} \mathrm{~N}$, ${ }^{20} \mathrm{Ne},{ }^{24} \mathrm{Mg},{ }^{32} \mathrm{~S}$, and ${ }^{40} \mathrm{Ca}$ ) with a quasi-infinite target was simulated using GEANT4. In all calculations the cylindrical target from natural uranium with a length of $160 \mathrm{~cm}$ and a radius of $60 \mathrm{~cm}$ was considered. The simulation was performed for beam energies from 0.3 to $10 \mathrm{GeV} /$ nucleon. The well studied part of the neutron spectrum below $20 \mathrm{MeV}$ was simulated using the parameterizations based on the experimental data ENDF (Evaluated Nuclear Data File). Special attention was paid to the simulation of inelastic hadron interactions.

It was demonstrated in [17] that GEANT4 is applicable for quantitative description of interaction of beams with intermediate energies (from hundreds $\mathrm{MeV}$ to several $\mathrm{GeV}$ ) with various heavy metal targets with a precision of $30 \%$. The comparative analysis of calculations using different codes, namely, GEANT4, MCNPX, and SHIELD [18] also demonstrated satisfactory agreement on a level of $30 \%$ for ion beam interaction with thick targets. Experimental data on distribution of fission reactions inside the extended uranium targets using $0.5-4 \mathrm{GeV} /$ nucleon deuteron beams $[7,14]$ agree within $30 \%$ with the results of GEANT4 simulation.

All ionization losses (including ionization losses of $\delta$ electrons and gamma conversion) for all charged particles, including primary beam particles and all secondary particles from successive nuclear reactions inside the target, namely, fission fragments, particles produced in multifragmentation, all produced particles and decaying charged particles until their complete stopping in the target (or until they reach the boundary and leave the bulk of the target), were summed for calculation of the energy deposited in the target $E_{d e p}$.

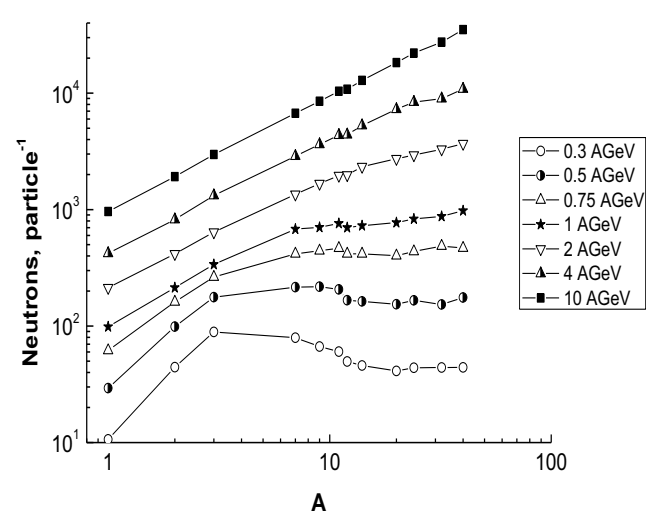

(a)

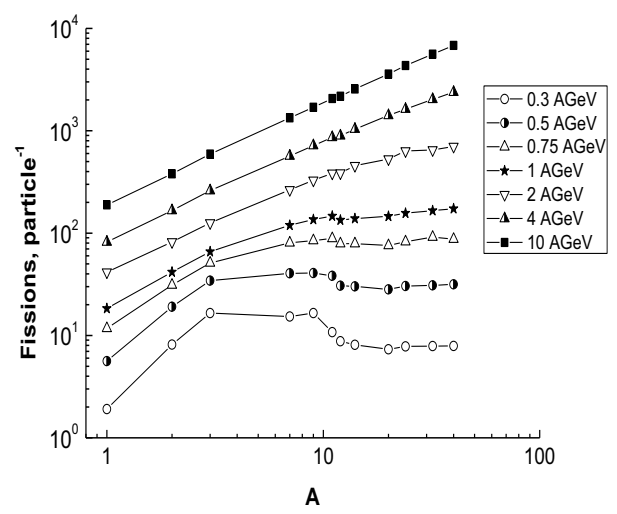

(b)

Figure 1. (a) Neutron yield and (b) number of fissions as functions of the beam ion mass number.

Figure 1 shows the simulated (a) total number of neutrons produced in the target and (b) the number of fissions in natural uranium normalized per one beam ion as functions of the mass number of beam ions for the set of energies in a range of $0.3-10 \mathrm{GeV} /$ nucleon.

Figure 2 shows the integral energy deposited Edep in the considered target for different beam energies as a function of mass number of the beam particles. 
The energy deposition in the quasi-infinite target for $10 \mathrm{GeV} /$ nucleon approaches the asymptotic dependence on the mass number of beam ions from protons to ${ }^{40} \mathrm{Ca}$. Note that the transition to the asymptotic (integral deposited energy proportional to the ion mass number) depends on the beam ion energy. For $1 \mathrm{GeV} /$ nucleon this asymptotic is applicable for light nuclei from protons to lithium, and for $2 \mathrm{GeV} /$ nucleon, up to carbon ions.

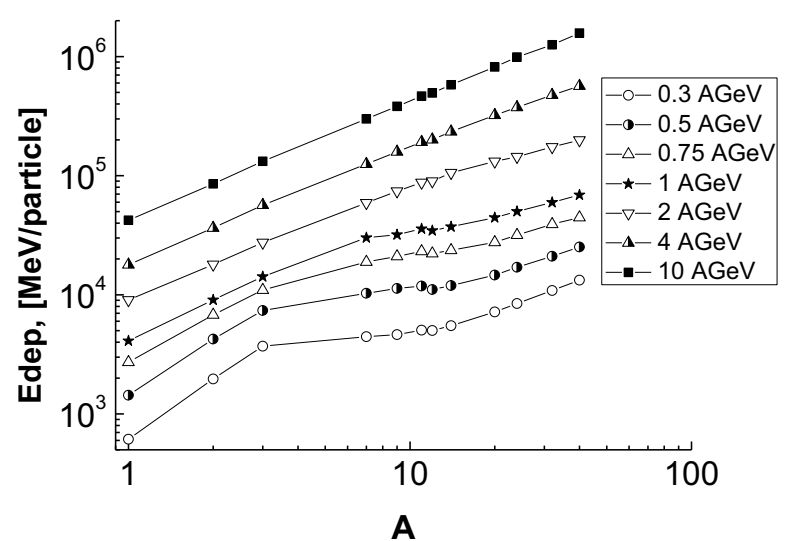

Figure 2. Integral energy deposition per one beam ion as a function of mass number of the incident ion.

The uranium fission channel makes the main contribution into the energy amplification factor due to nuclear transformations. It was demonstrated in [15] that for uranium and other actinides the ratio of the fission cross section and the total inelastic cross section is $\sim 90 \%$ for incident proton energy below $1 \mathrm{GeV}$, and this ratio smoothly tends to $40 \%$ for proton energies above $10 \mathrm{GeV}$. Taking into account the known energy of one uranium nucleus fission event equal to $190 \mathrm{MeV}$, the relative contribution into the energy deposition in the target from fission reactions as a function of energy for protons and several light nuclei is shown in figure 3.

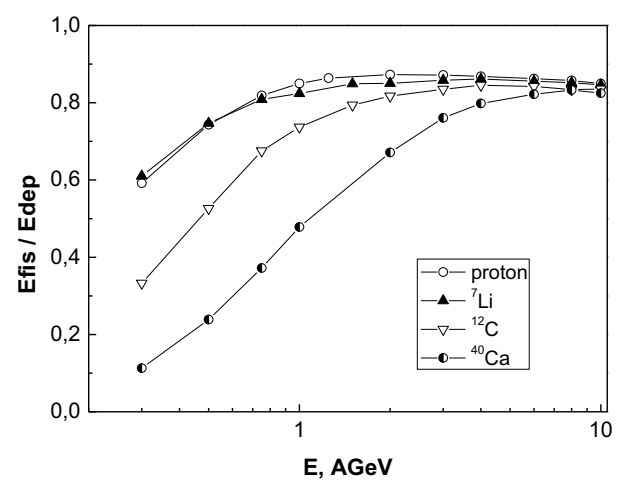

Figure 3. Relative contribution of energy deposited in the target from uranium fission reactions as a function of kinetic energy per nucleon of incident ions.

The simulations shown in figure 2 demonstrate that the $0.5 \mathrm{GeV} /$ nucleon deuteron beam has the same energy deposition as the $1 \mathrm{GeV}$ proton beam, and the $0.5 \mathrm{GeV} /$ nucleon ${ }^{7} \mathrm{Li}$ beam is equivalent to the $3 \mathrm{GeV}$ proton beam. 
In this study, for the purpose of comparative analysis of energy consumption in different types of accelerators and accelerated ions, we assume that the energy spent for maintaining the magnetic field per unit length is the same for all cyclic accelerators. The energy spent for maintaining the magnetic field in a synchrotron is $E_{\text {spent }} \sim A P / Z$, where $Z$ is the charge, $A$ is the mass number, and $P$ is the momentum of the accelerated ion. For an isochronous cyclotron, we have $E_{\text {spent }} \sim(A P / Z)^{2}$. We assume that for a linear accelerator the spent energy is $E_{\text {spent }} \sim A E_{k} / Z$, where $E_{k}$ is the kinetic energy per nucleon of the accelerated ion.

Let us consider the relative efficiency $\varepsilon_{r}$ for ion 2, in comparison with ion 1 for different accelerator types,

$$
\varepsilon_{r}=\frac{E_{\text {dep } 2}}{E_{\text {dep } 1}} \frac{E_{\text {spent } 1}}{E_{\text {spent } 2}} .
$$

Here, $E_{\text {dep } 2}$ and $E_{\text {depl }}$ are the energies deposited in the target for ions 1 and 2 (see figure 2), and $E_{\text {spent } 1}$ and $E_{\text {spent } 2}$ are the energy expenses according to the above assumptions for each accelerator type.

Figure 4 shows the relative efficiencies $\varepsilon_{r}$ of energy deposition in the quasi-infinite uranium target as functions of energy and type of accelerated ions. All dependences are normalized to the $1 \mathrm{GeV}$ proton beam (ion 1).

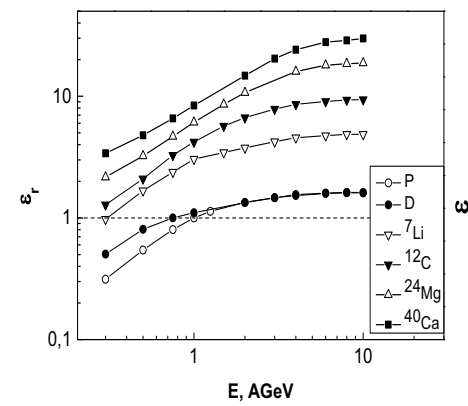

a)

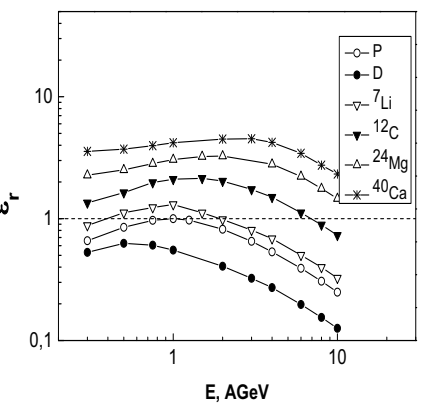

b)

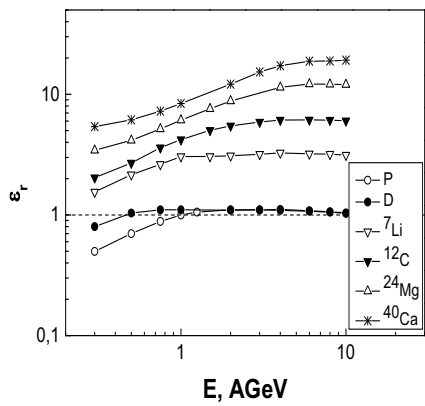

c)

Figure 4. Relative efficiency of ion energy deposition with respect to $1 \mathrm{GeV}$ protons for beams accelerated in a (a) synchrotron, (b) cyclotron, and (c) linear accelerator.

It can be seen from figure 4 that the optimal proton energy for a synchrotron is $\sim 3$ $\mathrm{GeV}$, for a linear accelerator, $\sim 1-1.5 \mathrm{GeV}$, and for a cyclotron, $\sim 1 \mathrm{GeV}$. The energy efficiency of light ions is considerably higher than that of protons. Noting this energy efficiency, the optimal energy range shifts from $1 \mathrm{GeV} /$ nucleon for ${ }^{7} \mathrm{Li}$ to $4-6 \mathrm{GeV} /$ nucleon for ${ }^{40} \mathrm{Ca}$.

It should also be noted that the $0.5 \mathrm{GeV} /$ nucleon ${ }^{7} \mathrm{Li}$ beam is equivalent from the point of view of energy deposition to the $2 \mathrm{GeV}$ proton beam (or $0.75 \mathrm{GeV} /$ nucleon ${ }^{7} \mathrm{Li}$ beam is equivalent to the $3 \mathrm{GeV}$ proton beam). This allows one to obtain the equivalent energy with the same or higher efficiency using an accelerator with the twice as small size using the ${ }^{7} \mathrm{Li}$ beam, as compared to the use of the proton beam.

\section{Conclusions}

The optimal proton energy for a synchrotron is $\sim 3 \mathrm{GeV}$, for a linear accelerator, $\sim 1-1.5$ $\mathrm{GeV}$, and for a cyclotron, $\sim 1 \mathrm{GeV}$.

The optimal accelerator energy depends on the type of accelerated ion; thus, for carbon the optimal energy is $2 \mathrm{GeV} /$ nucleon, and for ${ }^{40} \mathrm{Ca}$ ions, $4 \mathrm{GeV} /$ nucleon. 
In terms of energy deposition, $0.5 \mathrm{GeV} /$ nucleon ${ }^{7} \mathrm{Li}$ beam is equivalent to $2 \mathrm{GeV}$ protons. This is especially topical for the choice and optimization of the accelerator parameters for an accelerator driven fast neutron reactor.

The experiments with carbon beams [14] indicate that it is necessary to develop and specify the models for description of the experimentally observed enhancement of the hard part of the neutron spectrum in an energy range of $0.5-4 \mathrm{GeV} /$ nucleon. The forthcoming experiments with light ion beams at Nuclotron are topical.

We thank V. I. Yurevich, N. M. Sobolevskii, A. I. Malakhov, A. D. Kovalenko, and A. V. Butenko for helpful discussions.

\section{References}

1. G. A. Bartolomeev, Intense Neutron Source, CONF-660, 925 (Santa Fe, 1963)

2. V. P. Dmitrievskii, Phys. Part. Nucl. 28(3), 815 (1997)

3. V. N. Mikhailov, JINR Rapid Commun. 6[80]-96, 17 (1996)

4. C. Rubbia et al., CERN/AT/93-47 (1993)

5. K. Ismailov, M. Saito, H. Sagara, K. Nishihara, Progress in Nucl. Energy 53, 925 (2011)

6. J. Adam, et al. Collaboration E\&T-RAW (Energy and Transmutation RAW) JINR Preprint, E1-2010-61 (2010)

7. A. A. Baldin et al., Collaboration E\&T-RAW (Energy and Transmutation RAW), Phys. Part. Nucl. Lett. 8(6), 606 (2011)

8. D. G. Koshkarev, N. M. Sobolevskii, A. V. Barkhudaryan, Atomic Energy, 105(3), 225 (2008)

9. V. F. Batyaev et al., Atomic Energy, 104(4), 319 (2008)

10. S. R. Hashemi-Nezhad, W. Westmeier, M. Zamani-Valasiadou, B. Thomauske, R. Brandt, Annals of Nuclear Energy 38, 1144 (2011)

11. V. I. Yurevich, Phys. Part. Nucl. 41(5), 778 (2010)

12. V. Pronskikh, N. V. Mokhov, I. Novitski, and S. I. Tyutyunikov, 12th Meeting on Shielding Aspects of Accelerators, Targets and Irradiation Facilities (SATIF-12) (FNAL, Batavia, Illinois, USA, 2014)

13. W. Furman et al. Collaboration E\&T-RAW (Energy and Transmutation RAW), XXI International Baldin Seminar on High Energy Physics Problems, (JINR, Dubna, Russia, 2012)

14. M. Artiushenko, A. Baldin, V. Chilap, A. Chinenov, W. Furman, K. Husak, Yu. Petrusenko, V. Sotnikov, S. Tyutyunnikov, V. Voronko, and I. Zhuk, XXII International Baldin Seminar of High Energy Physics Problem, (JINR, Dubna, Russia, 2014)

15. V. I. Yurevich, V. A. Nikolaev, R. M. Yakovlev, and A. N. Sosnin, Phys. At. Nucl. 65(8), 1383 (2002)

16. M. Paraipan, A. A. Baldin, M. G. Kadykov, and S. I. Tyutyunikov, XXI International Baldin Seminar on High Energy Physics Problems, (JINR, Dubna, Russia, 2012)

17. A. A. Baldin, A. I. Berlev, I. V. Kudashkin, G. Mogildea, M. Mogildea, M. Paraipan, and S. I. Tyutyunnikov, Phys. Part. Nucl. Lett. 13(2), 249 (2016)

18. A. Krylov, M. Paraipan, N. Sobolevsky, G. Timoshenko, and V. Tretyakov, Phys. Part. Nucl. Lett. 11(4), 549 (2014) 